OPEN ACCESS

Edited by:

Miklos Fuzi,

Semmelweis University, Hungary

Reviewed by:

Noton Kumar Dutta,

Johns Hopkins University,

United States

Vishvanath Tiwari,

Central University of Rajasthan, India

*Correspondence:

Jiyue Cao

caojiyue2@163.com

Yinsheng Qiu

qiuyinsheng6405@163.com

Specialty section:

This article was submitted to

Antimicrobials, Resistance and

Chemotherapy,

a section of the journal

Frontiers in Microbiology

Received: 30 March 2017 Accepted: 18 October 2017 Published: 01 November 2017

Citation:

Yang B, Lei Z, Zhao Y, Ahmed S,

Wang C, Zhang S, Fu S, Cao J and

Qiu Y (2017) Combination

Susceptibility Testing of Common

Antimicrobials in Vitro and the Effects

of Sub-MIC of Antimicrobials on

Staphylococcus aureus Biofilm

Formation. Front. Microbiol. 8:2125.

doi: 10.3389/fmicb.2017.02125

\section{Combination Susceptibility Testing of Common Antimicrobials in Vitro and the Effects of Sub-MIC of Antimicrobials on Staphylococcus aureus Biofilm Formation}

\author{
Bing Yang ${ }^{1,2}$, Zhixin Lei ${ }^{1,2}$, Yishuang Zhao ${ }^{1}$, Saeed Ahmed ${ }^{2}$, Chunqun Wang ${ }^{1}$, \\ Shishuo Zhang ${ }^{1}$, Shulin $\mathrm{Fu}^{3}$, Jiyue $\mathrm{Cao}^{1,2 *}$ and Yinsheng Qiu ${ }^{3 *}$
}

\begin{abstract}
${ }^{1}$ Department of Veterinary Pharmacology, College of Veterinary Medicine, Huazhong Agricultural University, Wuhan, China, ${ }^{2}$ National Reference Laboratory of Veterinary Drug Residues and Key Laboratory for Detection of Veterinary Drug Residues, Huazhong Agricultural University, Wuhan, China, ${ }^{3}$ School of Animal Science and Nutritional Engineering, Wuhan Polytechinic University, Wuhan, China
\end{abstract}

The current study was conducted to evaluate the antibacterial combination efficacies, and whether the sub-inhibitory concentrations (sub-MIC) of antibiotics can influent on the biofilm formation of $S$. aureus. The minimum inhibitory concentration (MIC) of common antibacterial drugs was determined in vitro against clinical isolates of Staphylococcus aureus (S. aureus), Escherichia coli (E. coli), and Pasteurella multocida (P. multocida) alone and in combination with each other by using the broth microdilution method and the checkerboard micro-dilution method analyzed with the fractional inhibitory concentration index $(\mathrm{FICl})$, respectively. Regarding these results, antibacterial drug combinations were categorized as synergistic, interacting, antagonistic and indifferent, and most of the results were consistent with the previous reports. Additionally, the effects of sub-MIC of seven antimicrobials (kanamycin, acetylisovaleryltylosin tartrate, enrofloxacin, lincomycin, colistin sulfate, berberine, and clarithromycin) on S. aureus biofilm formation were determined via crystal violet staining, scanning electron microscopy (SEM) and real-time PCR. Our results demonstrate that all antibiotics, except acetylisovaleryltylosin tartrate, effectively reduced the $S$. aureus biofilm formation. In addition, real-time reverse transcriptase PCR was used to analyze the relative expression levels of $S$. aureus biofilm-related genes such as sarA, fnbA, rbf, $\operatorname{Irg} A, \operatorname{cid} A$, and eno after the treatment at sub-MIC with all of the six antimicrobials. All antibiotics significantly inhibited the expression of these biofilm-related genes except for acetylisovaleryltylosin tartrate, which efficiently up-regulated these transcripts. These results provide the theoretical parameters for the selection of effective antimicrobial combinations in clinical therapy and demonstrate how to correctly use antibiotics at sub-MIC as preventive drugs.

Keywords: antimicrobial, sub-MIC, morphological observation, biofilm formation 


\section{INTRODUCTION}

In recent years, there has been an increasing tendency regarding the misuse of antimicrobials in clinical veterinary treatments. The widespread abuse of antibacterial drugs for the treatment of bacterial infections has led to the emergence and spread of drug-resistant. Presently, treatment with antimicrobials alone is not guaranteed to cure the serious and mixed infections. There is increasing clinical evidence on the base of combination therapy of antimicrobials are a valuable treatment option against the mixed or multidrug-resistant bacterial infections (Bharat et al., 2016; Stein et al., 2016; Wei et al., 2016). Combinations of antibacterial agents are a reasonable way to enhance the antibacterial action of drugs, especially for serious and mixed infections. In vitro, the activities of drugs combination can be determined by a checkerboard micro-dilution method or timekill experiments with static antibiotic concentrations, which are usually used to evaluate susceptibility to combinations of antibiotics (Hall et al., 1983). According to standard definitions, the effects of antibiotic combinations are divided into synergy, interaction, indifference and antagonism. Many previous studies of antibiotic combinations have demonstrated that the antimicrobial effects of beta-lactams in combination with macrolides are antagonistic, but the reports are conflicting (Yoshioka et al., 2016; Lee et al., 2017). A study by Lodise et al. reported that beta-lactams were used in combination with macrolides in the treatment of community-acquired pneumonia (Lodise et al., 2007). In this study, the effects of common antibiotic combinations on S. aureus, E. coli, and P. multocida, representing both Gram-positive and Gram-negative bacteria, were investigated in vitro. In addition, quinolones, rifamycins, lincosamides and an extract of Chinese medicine were included as experimental drugs in this report.

Biofilms are a matrix composed of polymeric substances, DNA, and proteins that form a protective layer around bacteria (Donlan, 2002; Cue et al., 2009; Lee et al., 2014). Previous studies had been reported that biofilms can reduce the susceptibility of bacteria to antimicrobials (Stewart and Franklin, 2008). The development of bacterial biofilms can provide an extracellular barrier against the antimicrobial agents (Hsu et al., 2011). A review published by Ranita Roy et al has been reported the mechanism of $S$. aureus biofilm formation with the reference to different models and various methods used for anti-biofilm formation. In addition, various anti-biofilm molecules and their action mechanisms which may include herbal active compounds, chelating agents, peptide antibiotics, lantibiotics, and synthetic chemical compounds have been listed and introduced in this review (Roy et al., 2017). Some studies have demonstrated the antimicrobial agents at concentrations below their minimum inhibitory concentration (sub-MIC) have a clear influence on the biofilm formation (Majtán et al., 2008; Dong et al., 2012; El Haj et al., 2016). Some researchers have indicated that antimicrobial agents may inhibit or induce the bacterial biofilm formation at sub-MIC to avoid the chronic infection. For instance, Vishvanath Tiwari et al. have discovered and reported that the Actinidia deliciosa extract can reduce the production of exopolysaccharides (EPS), proteins and extracellular-DNA (e-DNA) to interference
Acinetobacter baumannii biofilm formation (Tiwari et al., 2017). In addition, the sub-MIC of antibiotics could result in morphological changes and changes in the relative expression levels of biofilm-related genes in bacterial strains.

In order to determine the most effective drug combinations remedy against bacterial infections, evaluating the efficacy of drug combinations and discussing the effect of sub-MIC of drugs on biofilm formation, the following trails have been carried out. These investigations are based on the original classification of antibiotics, adding quinolones, rifamycins, lincosamides and herbal extracts-berberine, study their interactions between drugs on clinical isolates of S. aureus, E. coli, and P. multocida, representing both Gram-positive and Gram-negative bacteria, with representative drugs including amoxicillin, ceftiofur, kanamycin, colistin sulfate, doxycycline, acetylisovaleryltylosin tartrate, florfenicol, sulfadimidine, enrofloxacin, rifampicin, berberine, and lincomycin. In addition, we studied the effects of sub-MIC antibiotics on clinical bacteria biofilm formation. Bacteria were screened for their ability to form biofilms under experimental conditions and isolates were used to study the effect of sub-MIC of drugs on biofilm formation via crystal violet staining, scanning electron microscopy (SEM) and real-time polymerase chain reaction (RT-PCR). These experiments provide theoretical knowledge for veterinary clinical use and define the optimal antimicrobial combinations. We also warn people that the use of sub-MIC for the biofilm formation must be based on a laboratory research results.

\section{MATERIALS AND METHODS}

\section{Bacterial Strains}

Clinically isolated strains of S. aureus (S. aureus Hb0206 isolated from pigs in Hubei, China), E. coli (E. coli Hd311 isolated from pigs in Hubei, China) and P. multocida (P. multocida 0625 isolated from pigs in Hubei, China) are provided by the microbiological lab of Huazhong Agriculture University. Escherichia coli $\mathrm{ATCC}_{25922}$ and Staphylococcus aureus $\mathrm{ATCC}_{25923}$ were chosen as quality control strains in all of the agent's susceptibility testing.

Escherichia coli and Staphylococcus aureus strains were cultured in $5 \mathrm{~mL}$ of nutrient broth at $37^{\circ} \mathrm{C}$ overnight. Then the E. coli strains were streaked from nutrient broth and grown on MacConkey agar plates at $37^{\circ} \mathrm{C}$ overnight. S. aureus strains were grown on nutrient agar plates at $37^{\circ} \mathrm{C}$ overnight. P. multocida strains were grown on TSA plate medium with 5\% fetal bovine serum and were cultured at $37^{\circ} \mathrm{C}$ overnight. All of the incubation above aims to separate and purify isolated strains. A single bacterial colony was used to inoculate $5 \mathrm{~mL}$ MuellerHinton (MH) broth and was incubated at $37^{\circ} \mathrm{C}$ overnight, and P. multocida strains should with $5 \%$ fetal bovine serum.

\section{Preparation of Antimicrobial Agents}

Kanamycin, doxycycline, acetylisovaleryltylosin tartrate, enrofloxacin, lincomycin, and colistin sulfate standards were obtained from the Wuhan Huisheng Biotechnology Group. Amoxicillin, ceftiofur, and rifampicin standards were purchased from Changfengda Commercial Trading Company (Wuhan, 
China). Florfenicol and sulfadimidine standards were purchased from the Biotechnology Research Institute (Beijing, China). The berberine standard was provided by the National Institutes for Food and Drug Control. Antibiotic stock solutions were prepared in advance and stored at $-20^{\circ} \mathrm{C}$. The concentration of all stock solutions was $5120 \mu \mathrm{g} / \mathrm{mL}$.

\section{Combination Susceptibility Testing}

Firstly, the MICs of each of the 12 drugs were determined in the three bacterial species by the broth microdilution method according to the Clinical and Laboratory Standards Institute (CLSI) M31-A3 guidelines (2012) (CLSI, 2008). The checkerboard micro-dilution method was used to test combination susceptibility of the 12 candidate antimicrobials in the three bacterial strains according to Pankey et al (Pankey and Ashcraft, 2009). Serial two-fold dilutions were prepared in broth ranging from $1 / 4$ to 16 -fold the MIC concentration for drug A and drug B, respectively. A total of $100 \mu \mathrm{L}$ of 8 to $1 / 4$-fold MIC concentration of drug A was added to row 1 and columns A-F, and then $50 \mu \mathrm{L}$ of 16 to $1 / 2$-fold MIC concentration of drug A was added to rows A-G and columns 2-7. A total of $100 \mu \mathrm{L}$ of $1 / 4$ to 8 -fold MIC concentration of drug $\mathrm{B}$ was added to row $\mathrm{H}$ and columns $2-7$, and then $50 \mu \mathrm{L}$ of $1 / 2$ to 16 -fold MIC concentration of drug $B$ was added to columns $2-7$ and rows A-G. A total of $200 \mu \mathrm{L}$ broth was added to column 1 and rows $\mathrm{H}$. And the checkerboard plates were inoculated with $10^{6}$ c.f.u. $/ \mathrm{mL}$ of strains and incubated at $37^{\circ} \mathrm{C}$ for $24 \mathrm{~h}$.

The FICI was calculated for each antimicrobial in each combination to evaluate the effect of the combination. The formulas used to calculate the FICI are as follows:

FICI of drug A = (MIC of drug A in combination) / (MIC of drug A alone)

FICI of drug B = (MIC of drug B in combination) / (MIC of drug $\mathrm{B}$ alone)

$\sum$ FICI $=$ FICI of drug A + FICI of drug B

A stringent definition was used to explain the effect of combination susceptibility of the drugs. Synergy was defined as $\sum$ FICI $\leq 0.5$. The interaction was defined as $0.5<\sum$ FICI $\leq 1$. Indifference was defined as $1<\sum$ FICI $\leq 2$. Antagonism was defined as $\sum$ FICI $>2$. All tests were repeated in triplicate.

\section{Selection of Bacteria with a Strong Ability to Form Biofilms}

The biofilm formation assays were performed by the crystal violet staining method using sterile 96-well polystyrene plates. Staphylococcus aureus, Escherichia coli, and Pasteurella multocida were used in the experiments. Bacterial strains were used to inoculate $5 \mathrm{~mL}$ of TSB after an overnight culture on TSA at $37^{\circ} \mathrm{C}$. The bacterial suspension was transferred to a 96-well polystyrene plate $(100 \mu \mathrm{L} /$ well $)$ at a concentration of $1.0 \times$ $10^{7} \mathrm{CFU} / \mathrm{mL}$. Media lacking bacteria was used as a negative control.

After a $24 \mathrm{~h}$ incubation period at $37^{\circ} \mathrm{C}$ without shaking, the content of each well was removed, and the wells were carefully washed two times with sterile distilled water. The plates were airdried in a ventilator and stained with $120 \mu \mathrm{L}$ of a $1 \% \mathrm{w} / \mathrm{v}$ crystal violet solution. Crystal violet was removed after 15 20 min and the wells were washed with $300 \mu \mathrm{L}$ of sterile distilled water to remove excess bacterial strains. The dye bound to the bacteria was dissolved thoroughly with $150 \mu \mathrm{L} 33 \%$ glacial acetic acid for $10 \mathrm{~min}$. The absorbance in each well was measured with a spectrophotometer at $630 \mathrm{~nm}\left(\mathrm{OD}_{630}\right)$.

The strains were classified into four different categories according to their biofilm-forming ability depending on their 2 $\mathrm{OD}_{\mathrm{c}}$ (the average OD of the blank well at threefold its standard deviation) of bacteria. The categories were delineated as follows: not able to form a biofilm $\left(\mathrm{OD}_{\mathrm{c}}<\mathrm{OD}<2 \mathrm{OD}_{\mathrm{c}}\right)$, weakly able to form a biofilm $\left(2 \mathrm{OD}_{\mathrm{c}}<\mathrm{OD}<4 \mathrm{OD}_{\mathrm{c}}\right)$, moderately able to form a biofilm $\left(4 \mathrm{OD}_{\mathrm{c}}<\mathrm{OD}<6 \mathrm{OD}_{\mathrm{c}}\right)$, and strongly able to form a biofilm $\left(6 \mathrm{OD}_{\mathrm{c}}<\mathrm{OD}\right)$.

\section{Screening for Optimum Conditions of Biofilm Formation}

Staphylococcus aureus was grown at $37^{\circ} \mathrm{C}$ in TSB as in previous studies. Strains that had a strong ability to form biofilms were cultured in TSB media at $37^{\circ} \mathrm{C}$ for $1-5$ days. Subsequently, crystal violet staining was used to optically screen for the best conditions for bacterial biofilm formation.

\section{Effect of Sub-MIC on Biofilm Formation with Crystal Violet Staining}

A strongly biofilm-forming isolate was selected to test the influence of sub-MIC of some antimicrobials on biofilm formation. The trail was performed in sterile 96-well polystyrene plates. The methods were the same as the selection test for identifying strong biofilm-forming isolates. The tested drugs were kanamycin, colistin sulfate, acetylisovaleryltylosin tartrate, enrofloxacin, berberine, lincomycin, and clarithromycin. The isolates were exposed to $1 / 2 \mathrm{MIC}, 1 / 4 \mathrm{MIC}, 1 / 8 \mathrm{MIC}, 1 / 16 \mathrm{MIC}$, 1/32 MIC, 1/64 MIC and 1/128 MIC supplemented in TSB at $37^{\circ} \mathrm{C}$ for $48 \mathrm{~h}$. Furthermore, wells lacking drugs and lacking bacteria in the same media served as the negative control and blank control, respectively. After incubation, biofilm formation was quantified by crystal violet staining. The tests were performed three times.

\section{Morphological Observations by SEM}

SEM was used to observe morphological variations in biofilm formation following treatment with antimicrobial agents. TSB was mixed with each antimicrobial agent at 1/2 MIC and was pipetted onto sterile culture dishes containing $S$. aureus. The same medium and inoculum, lacking agents, was used as a control. A sterile plastic coverslip was inserted into every dish. After overnight incubation at $37^{\circ} \mathrm{C}$, the coverslips were carefully washed twice with sterile PBS following guidelines of the microscopy core laboratory at Huazhong Agricultural University. $S$. aureus biofilm formation on the coverslips was observed with a HITACHI SU8010.

\section{Real-Time Polymerase Chain Reaction (RT-PCR)}

RT-PCR was used to analyze the relative expression of biofilmrelated genes such as $\operatorname{sar} A, f n b A, \operatorname{rbf}, \operatorname{lrg} A, \operatorname{cid} A$, and eno. The primer sequences are listed in Table 1. Overnight cultures 
TABLE 1 | Primers and sequences used in this study.

\begin{tabular}{|c|c|c|c|c|}
\hline Gene & Primer & Sequence $\left(5^{\prime} \rightarrow 3^{\prime}\right)$ & References & Accession \\
\hline \multirow[t]{2}{*}{ sarA } & sarA-F & TCTTGTTAATGCACAACAACGTAA & Zielinska et al., 2012; Abdelhady et al., 2014 & DQ414117.1 \\
\hline & sarA-R & TGTITGCTTCAGTGATTCGTIT & & \\
\hline \multirow[t]{2}{*}{$f n b A$} & $f n b A-F$ & GAAGAGCATGGTCAAGCACA & Lee et al., 2014 & KP096552 \\
\hline & $f n b A-R$ & ACGTCATAATTCCCGTGACC & & \\
\hline \multirow[t]{2}{*}{$r b f$} & $r b f-F$ & ACGCGTTGCCAAGATGGCATAGTCTT & Cue et al., 2009 & KR001974.1 \\
\hline & $r b f-R$ & AGCCTAATTCCGCAAACCAATCGCT & & \\
\hline \multirow[t]{2}{*}{$\operatorname{Irg} A$} & $\operatorname{Irg} A-\mathrm{F}$ & AGACGCATCAAAACCAGCACACT & Hsu et al., 2011 & U52961.1 \\
\hline & $\operatorname{Irg} A-\mathrm{R}$ & CCGGCTGGTACGAAGAGTAAGCC & & \\
\hline \multirow[t]{2}{*}{$\operatorname{cid} A$} & $\operatorname{cidA-F}$ & AGCGTAATTTCGGAAGCAACATCCA & Hsu et al., 2011 & AY58192.1 \\
\hline & $\operatorname{cid} A-\mathrm{R}$ & CCCTTAGCCGGCAGTATTGTTGGTC & & \\
\hline \multirow[t]{2}{*}{ eno } & eno-F & TGCCGTAGGTGACGAAGGTGGTT & Atshan et al., 2013 & KU295448.1 \\
\hline & eno-R & GCACCGTGTTCGCCTTCGAACT & & \\
\hline $16 S r$ & 16SrRNA-F & GAGGGTCATTGGAAACTGGA & Lee et al., 2014 & AH013003.2 \\
\hline$R N A$ & 16SrRNA-R & CATITCACCGCTACACATGG & & \\
\hline
\end{tabular}

of $S$. aureus were added to fresh TSB and grown at $37^{\circ} \mathrm{C}$ with shaking. The drugs kanamycin, lincomycin, enrofloxacin, colistin sulfate, acetylisovaleryltylosin tartrate and berberine were dissolved in TSB to $1 / 2 \mathrm{MIC}, 1 / 4 \mathrm{MIC}, 1 / 8 \mathrm{MIC}, 1 / 16$ $\mathrm{MIC}$, and 1/32 MIC. The mixture of TSB with antimicrobial agents at sub-MIC and $S$. aureus cultures were transferred into test tubes. Tubes lacking antibiotics were used as controls. All experimental and control tubes were incubated at $37^{\circ} \mathrm{C}$ overnight, then $S$. aureus cells were resuspended in lysostaphin (Sigma-Aldrich) and incubated at $37^{\circ} \mathrm{C}$ for $30 \mathrm{~min}$. Then total RNA was extracted by the TRIzol (Takara) method followed by RNase-free DNase (Takara) treatment to eliminate any remaining DNA. The levels of mRNA were determined by real-time quantitative reverse-transcription PCR with the Quant Script RT Kit (Takara) and a Real Master Mix (SYBR Green II) Kit. All samples were carried out in triplicate and three independent experiments were performed. Expression levels of the genes were normalized to $16 \mathrm{~S}$ rRNA. The changes in each transcript were determined by the $2^{-\Delta \Delta \mathrm{T}}$ method when compared to drug-free cells.

\section{Ethics Statement}

All bacterium experiments and experimental protocols used in this study were conducted in accordance with the Guide for the Care and Use of Laboratory Animals of Hubei Provincial Laboratory Animal Service Center (permit number SYXK 20130044) and approved by the Ethics Committee of Huazhong Agricultural University, Wuhan, China.

\section{Statistical Analysis}

The experimental data were analyzed using a one-way analysis of variance (ANOVA) by SPSS 22.0 and the statistical results are reported as a mean \pm standard deviation. Pairwise comparisons with differences of $P \leq 0.05$ were considered statistically significant; $P \leq 0.01$ was considered extremely significant.

\section{RESULTS}

\section{Combination Susceptibility Testing of 12 Kinds of Drugs}

Firstly, the MICs of 12 different drugs in three species of bacteria were determined according to the CLSI standard and within the scope of quality control. The results are shown in Table 2.

Results showed that the strain of $S$. aureus was sensitive to most of the used drugs and resistant to doxycycline, florfenicol and berberine. Escherichia coli was susceptible to ceftiofur, kanamycin, colistin sulfate, florfenicol and rifampicin while resistant to amoxicillin, doxycycline, acetylisovaleryltylosin tartrate, sulfadimidine, enrofloxacin, berberine, and lincomycin. P. multocida was susceptible to amoxicillin, ceftiofur, colistin sulfate, doxycycline, florfenicol and rifampicin while resistant to kanamycin, acetylisovaleryltylosin tartrate, sulfadimidine, enrofloxacin, berberine and lincomycin.

The combination susceptibility of these 12 antibiotics on the three strains was assigned synergistic, interacting, antagonistic and indifferent according to the FICI.

The effects of antibacterial combinations on S. aureus, our representative Gram-positive bacteria, are shown in Table 3A. Enrofloxacin in combination with ceftiofur showed an additive effect $(0.5<$ FICI $<1)$, as well as rifampin in combination with ceftiofur or doxycycline and berberine in combination with florfenicol or sulfadimidine. When enrofloxacin was combined with doxycycline, florfenicol or sulfadimidine, when rifampin was combined with kanamycin or florfenicol, or when lincomycin was combined with acetylisovaleryltylosin tartrate or colistin sulfate, we observed an antagonistic effect (FICI $>2$ ). The rest of the combinations had an indifferent effect $(1<$ FICI $<2)$. The FICI of berberine in combination with acetylisovaleryltylosin tartrate or ceftiofur was 1.5 , indicating that these combinations could be applied to reduce toxicity and economize costs with fewer doses. In the current study, the doxycycline, florfenicol and berberine showed high MIC values against $S$. aureus, which indicates that $S$. aureus strains 
TABLE 2 | MICs of 12 different drugs in three species of bacteria.

\begin{tabular}{lccc}
\hline Formulation & \multicolumn{3}{c}{ MIC $(\boldsymbol{\mu} \mathbf{g} / \mathbf{m L})$} \\
\cline { 2 - 4 } & S. ureus & E. coli & P. multocida \\
\hline Amoxicillin & $1 / 8$ & 32 & $1 / 2$ \\
Ceftiofur & 2 & $1 / 8$ & $1 / 16$ \\
Kanamycin & 8 & 2 & 16 \\
Colistin Sulfate & 1 & $1 / 8$ & 1 \\
Doxycycline & 32 & 32 & 1 \\
A-Tartrate & 2 & 128 & 128 \\
Florfenicol & 64 & 2 & 1 \\
Sulfadimidine & 8 & 16 & 16 \\
Enrofloxacin & 0.5 & 16 & 16 \\
Rifampicin & 8 & 4 & $1 / 2$ \\
Berberine & 128 & 512 & 32 \\
Lincomycin & 1 & 512 & 512
\end{tabular}

A-Tartrate stands for acetylisovaleryltylosin tartrate.

are resistant to these agents. Doxycycline in combining with berberine and florfenicol displayed the indifferent effects, while the berberine in combination with florfenicol showed an interactive effect. The synergistic effect was observed for the combination of acetylisovaleryltylosin tartrate and colistin sulfate against $S$. aureus, while sulfadimidine in combination with enrofloxacin showed an antagonistic effect against $S$. aureus. Notably, the isolate of $S$. aureus used in present study is sensitive to acetylisovaleryltylosin tartrate, colistin sulfate, sulfadimidine and enrofloxacin.

The effects of antibacterial combinations on $P$. multocida (Table 3B) and E. coli (Table 3C), representative Gram-negative bacteria, are shown as follows. The FICI was 1.5, which suggested that they can be used together with fewer doses. Enrofloxacin in combination with ceftiofur, colistin sulfate, sulfamethazine or berberine showed a synergistic and additive effect, while enrofloxacin with florfenicol or rifampicin exhibited an antagonistic and indifferent effect. Rifampicin in combination with colistin sulfate, doxycycline, florfenicol or berberine showed an additive effect, while rifampicin in combination with kanamycin, lincomycin or enrofloxacin showed an antagonistic and indifferent effect. Berberine with amoxicillin or florfenicol had an indifferent effect, while all others showed an additive and synergistic effect. Lincomycin with colistin sulfate or sulfamethazine showed a synergistic and additive effect, while lincomycin with amoxicillin, kanamycin, acetylisovaleryltylosin tartrate, florfenicol or rifampin exhibited an antagonistic effect. The rest of the combinations had an indifferent effect. Besides, the combination of berberine and doxycycline resulted in an interactive effect in vitro against $E$. coli that is resistant to the individual drugs. Similarly, the combination of acetylisovaleryltylosin tartrate and berberine showed an interactive effect against $P$. multocida that is resistant to the individual drugs. The synergistic effect was observed when ceftiofur was combined with colistin sulfate and the antagonistic effect has been observed when ceftiofur was combined with florfenicol against $E$. coli which is sensitive to all individual drugs.
Amoxicillin in combination with ceftiofur showed a synergistic effect against $P$. multocida that is resistant to the individual drugs. In contrast, the combination of amoxicillin and doxycycline showed an antagonistic effect against $P$. multocida that is resistant to doxycycline and amoxicillin.

\section{Bacterial Strains with a Strong Ability to Form Biofilms}

Biofilm formation assays were performed using the crystal violet staining method. The results are shown in Table 4.

An isolate of $S$. aureus that had an $\mathrm{OD}_{630}$ greater than $6 \mathrm{OD}_{\mathrm{c}}$ was considered to be a strong biofilm-forming strain. Isolates of E. coli and P. multocida that had $2 \mathrm{OD}_{\mathrm{c}}<\mathrm{OD}<4 \mathrm{OD}_{\mathrm{c}}$ or $\mathrm{OD}_{\mathrm{c}}<$ $\mathrm{OD}<2 \mathrm{OD}_{\mathrm{c}}$ were considered to be weak biofilm-forming strains or unable to form biofilms, respectively. S. aureus was used as the experimental strain.

\section{Optimizing Conditions for Biofilm Formation}

Previous studies have demonstrated that stains of $S$. aureus grow well at $37^{\circ} \mathrm{C}$ in tryptic soy broth (TSB). The culture time was identified through experimental means. The results are shown in Table 5. There was evidently biofilm formation after 1 day of culture at $37^{\circ} \mathrm{C}$, and biofilm formation reached its peak after 3 days of culture as determined by $\mathrm{OD}_{630}$ measurements. The difference between biofilm formation after 1, 4, and 5 days was not significant, and there was no significant difference between 2 and 3 days $(P>0.05)$. But there were significant differences between 1,4 , or 5 days and 2 or 3 days $(P \leq 0.05)$ Table 5 . According to the experimental data and statistical analysis, the optimal experimental conditions for biofilm formation were to culture for 2 days in $\mathrm{TSB}$ at $37^{\circ} \mathrm{C}$.

\section{Effect of Sub-MIC on Biofilm Formation with Crystal Violet Staining}

The biofilm formation of $S$. aureus treated with sub-MIC of different kinds of drugs was determined using crystal violet staining. Values were expressed as means of $\mathrm{OD}_{630} \pm$ standard deviations. Details of the results are shown in Figure 1.

Staphylococcus aureus biofilms incubated with kanamycin were significantly inhibited compared to the control $(P \leq 0.05)$, and the difference between each concentration tested was not significant. These results indicated that the different concentrations of kanamycin used are irregular in their ability to influence the inhibition of biofilm formation.

The levels of biofilm formation for $S$. aureus treated with 1/128 of the MIC of enrofloxacin exhibited no significant inhibition relative to the control $(P>0.05)$ but had a significant difference at other concentrations (1/64 1/2 MIC). Inhibition was positively correlated with the concentration of enrofloxacin at $1 / 16 \sim 1 / 2$ MIC.

Biofilm formation of $S$. aureus during treatment with berberine was significantly inhibited at concentrations of $1 / 8 \sim 1 / 2$ MIC compared to the control $(P \leq 0.05)$, and there was no significant difference between $1 / 128 \sim 1 / 16$ MIC treatments and the drug-free group $(P>0.05)$. But the difference between 
TABLE 3A | The FICl of 12 kinds of antibiotic against S. aureus.

\begin{tabular}{|c|c|c|c|}
\hline Agent $A$ and $B$ in combination & Effect on S. aureus & Agent $A$ and $B$ in combination & Effect on S. aureus \\
\hline amoxicillin+ceftiofur & Interaction & colistin sulfate+doxycycline & Synergy \\
\hline amoxicillin+kanamycin & Interaction & colistin sulfate+florfenicol & Interaction \\
\hline amoxicillin+colistin sulfate & Interaction & colistin sulfate+sulfadimidine & Interaction \\
\hline ceftiofur+kanamycin & Interaction & doxycycline+rifampine & Interaction \\
\hline ceftiofur+colistin sulfate & Interaction & A-Tartrate +florfenicol & Synergy \\
\hline ceftiofur+sulfadimidine & Interaction & A-Tartrate +sulfadimidine & Synergy \\
\hline ceftiofur+enrofloxacin & Interaction & florfenicol+sulfadimidine & Interaction \\
\hline ceftiofur+rifampine & Interaction & florfenicol+Berberine & Interaction \\
\hline kanamycin+colistin sulfate & Interaction & sulfadimidine+berberine & Interaction \\
\hline kanamycin+sulfadimidine & Interaction & & \\
\hline
\end{tabular}

TABLE 3B | The FICl of 12 kinds of antibiotic against $P$. multocida.

\begin{tabular}{|c|c|c|c|}
\hline Agent $A$ and $B$ in combination & Effect on $P$. multocida & Agent $A$ and $B$ in combination & Effect on $P$ multocida \\
\hline amoxicillin+ceftiofur & Synergy & colistin sulfate+rifampine & Interaction \\
\hline amoxicillin+kanamycin & Interaction & colistin sulfate+berberine & Synergy \\
\hline amoxicillin+colistin sulfate & Interaction & doxycycline+ A-Tartrate & Synergy \\
\hline ceftiofur+kanamycin & Synergy & doxycycline+sulfadimidine & Interaction \\
\hline ceftiofur+colistin sulfate & Interaction & doxycycline+rifampine & Interaction \\
\hline ceftiofur+sulfadimidine & Interaction & doxycycline+berberine & Interaction \\
\hline ceftiofur+enrofloxacin & Interaction & A-Tartrate+florfenicol & Interaction \\
\hline ceftiofur+berberine & Interaction & A-Tartrate +berberine & Synergy \\
\hline kanamycin+colistin sulfate & Interaction & florfenicol+sulfadimidine & Interaction \\
\hline kanamycin+doxycycline & Interaction & sulfadimidine+enrofloxacin & Interaction \\
\hline kanamycin+florfenicol & Interaction & sulfadimidine+berberine & Interaction \\
\hline colistin sulfate+doxycycline & Interaction & enrofloxacine+berberine & Interaction \\
\hline colistin sulfate+florfenicol & Interaction & rifampine+berberine & Interaction \\
\hline colistin sulfate+sulfadimidine & Interaction & & \\
\hline
\end{tabular}

TABLE $3 \mathbf{C}$ | The $\mathrm{FICl}$ of 12 kinds of antibiotic against E. coli.

\begin{tabular}{|c|c|c|c|}
\hline Agent $A$ and $B$ in combination & Effect on E. coli & Agent $A$ and $B$ in combination & Effect on E. coli \\
\hline amoxicillin+ceftiofur & Interaction & colistin sulfate+berberine & Interaction \\
\hline amoxicillin+kanamycin & Synergy & colistin sulfate+lincomycin & Synergy \\
\hline amoxicillin+colistin sulfate & Interaction & doxycycline+A-Tartrate & Interaction \\
\hline ceftiofur+kanamycin & Synergy & doxycycline+berberine & Interaction \\
\hline ceftiofur+berberine & Synergy & A-Tartrate+florfenicol & Interaction \\
\hline kanamycin+colistin sulfate & Interaction & A-Tartrate+sulfadimidine & Interaction \\
\hline kanamycin+sulfadimidine & Interaction & A-Tartrate + berberine & Interaction \\
\hline kanamycin+berberine & Interaction & florfenicol+rifampine & Interaction \\
\hline colistin sulfate+doxycycline & Interaction & sulfadimidine+enrofloxacin & Interaction \\
\hline colistin sulfate+doxycycline & Synergy & sulfadimidine+berberine & Interaction \\
\hline colistin sulfate+sulfadimidine & Interaction & sulfadimidine+lincomycin & Interaction \\
\hline colistin sulfate+enrofloxacin & Synergy & enrofloxacine+berberine & Interaction \\
\hline colistin sulfate+rifampine & Interaction & rifampine+berberine & Interaction \\
\hline
\end{tabular}

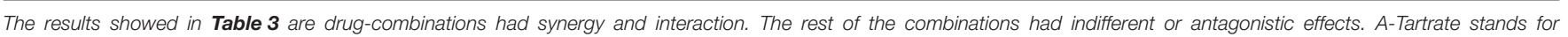
acetylisovaleryltylosin tartrate. 
TABLE $4 \mid \mathrm{OD}_{630}$ of 3 isolates.

\begin{tabular}{ccccc}
\hline Isolate & Control group & S. aureus & E. coli & Pasteurella \\
\hline $\mathrm{OD}_{630}$ & $0.052 \pm 0.001$ & $0.432 \pm 0.01^{\star \star}$ & $0.124 \pm 0.004^{\star}$ & $0.061 \pm 0.001$
\end{tabular}

$\mathrm{OD}_{630}$ means the absorbance of biofilm was measured with a spectrophotometer at $630 \mathrm{~nm}$. "Represent statistical significance ( $p \leq 0.05)$, " Represent extreme significance $(p \leq 0.01)$.

1/8 MIC treatment and 1/2 MIC treatment was not significant $(P>0.05)$.

Biofilm formation in $S$. aureus incubated with $1 / 16 \sim 1 / 2$ MIC lincomycin was significantly inhibited relative to the drug-free group $(P \leq 0.05)$, and the inhibitory effects were not significant between groups. The difference between other concentrations and the control were not significant $(P>0.05)$, indicating that biofilm growth was not affected by these concentrations of lincomycin.

Colistin sulfate at $1 / 4$ and $1 / 2$ MIC had a significant inhibitory effect on biofilm formation compared to the control $(P \leq 0.05)$, and the difference among groups was not statistically significant. The difference between lower concentrations (1/128 1/8 MIC) of colistin sulfate and the drug-free group was not statistically significant $(P>0.05)$ at inhibiting biofilm formation.

Biofilm formation was observably enhanced at $1 / 32 \sim 1 / 2$ MIC of acetylisovaleryltylosin tartrate, revealing that acetylisovaleryltylosin tartrate at these concentrations significantly induced biofilm formation relative to the control $(P \leq 0.05)$. The inducing effect of acetylisovaleryltylosin tartrate on biofilm formation was in agreement with changes at $1 / 32 \sim 1 / 4$ MIC. But the inducing effect was not significantly different when compared to the drug-free group at $1 / 64 \mathrm{MIC}(P>0.05)$. Thus, a lower dose of the drug may be the primary factor responsible for the decrease in its inducing effect.

To further confirm the effects of macrolides on $S$. aureus biofilm formation, we evaluated the action of clarithromycin on biofilm growth in vitro. The treated isolate exhibited a significant reduction $(P \leq 0.05)$ in biofilm formation for $1 / 64 \sim 1 / 2$ MIC clarithromycin in comparison with an isolate of drugs-free incubation. And the biofilm formation treated with a lower dose of clarithromycin was not significantly inhibited compared with drug-free groups. The results are shown in Figure 2.

\section{Morphological Observations by SEM}

Morphology of the $S$. aureus biofilm on the surface of coverslips was observed using SEM at 2000X magnification. SEM results were consistent with those from our crystal violet staining observations. Images of biofilms formed by $S$. aureus in the presence of sub-MIC of antibiotics are shown in Figure 3.

\section{Real-Time Polymerase Chain Reaction (RT-PCR)}

To determine whether the influence of antibiotics was manifested at the transcriptional level, total RNA was isolated from $S$. aureus following treatment at sub-MIC with antibiotics. The transcript levels for all biofilm-related genes are shown in Figure 4. In $S$. aureus strains, the down-regulation of all genes was observed by RT-PCR at sub-MIC treatments of kanamycin, lincomycin, enrofloxacin, berberine, and colistin sulfate. But the acetylisovaleryltylosin tartrate treatment group showed up-regulation for all genes. RT-PCR results showed that the down-regulation of all biofilm-related genes following berberine treatment was significant at $1 / 8 \sim 1 / 2$ MIC compared to the other groups $(P \leq 0.01$; Figure 4A. The lincomycin treatment group exhibited a significant decrease in the relative expression levels of all genes, except for $f n b A$, following treatment with 1/32 1/16 MIC lincomycin $(P \leq 0.01$; Figure 4B. The genes of $f n b A, \operatorname{rbf}, \operatorname{lrg} A$, and $\operatorname{cid} A$ were inhibited significantly following treatment with kanamycin at all sub-MIC, although the genes sarA and eno were not significantly different at 1/32 MIC (Figure 4C. Transcription levels for all genes were markedly decreased following treatment with enrofloxacin at sub-MIC $(P \leq 0.01)$, although the relative expression level of cidA was not significantly different at $1 / 32$ MIC of enrofloxacin (Figure 4D). Similar to the enrofloxacin treatment group, the colistin sulfate treatment group showed significant inhibition in the relative expression levels of all genes except for cidA (Figure 4E). Except for cidA, the transcript levels for all genes in biofilms treated with acetylisovaleryltylosin tartrate at subMIC had increased significantly, and at 1/2 MIC were extremely elevated (Figure 4F). And to assess the effects of macrolides with different member-ring on $S$. aureus biofilm formation, an addition assay that clarithromycin influent on biofilm formation was investigated to compare with acetylisovaleryltylosin tartrate treatment group.

\section{DISCUSSION}

\section{Combination Susceptibility Testing of 12 kinds of Drugs against Three Bacterial Isolates}

Existing knowledge about antibiotic combination therapy categorizes the effects of drug combinations as synergistic, interacting, indifferent and antagonistic. In vitro, the FICI obtained by the checkerboard dilution method can be usually used for the evaluation of antibiotic combinations (Pankey and Ashcraft, 2005). In the current study, a checkerboard microdilution method was used to analyze the antimicrobial activity of 12 kinds of drugs in alone and in combination with others against $S$. aureus, E. coli, and P. multocida to determine the value of combining conventional antibiotics. The results were consistent with previous studies.

At present, the effect of using antibiotics targeting the replication phase in combination with antibiotics targeting the stationary phase is controversial. Our results revealed that the combination of these two drugs types exhibited indifference and antagonism against $S$. aureus, and the most combinations exhibited the indifference and antagonism against $E$. coli and P. multocida. Macrolide antibiotics have a strong ability to disrupt the biofilms; thus macrolides are often used in conjunction with $\beta$-lactams or fluoroquinolones to enhance the ability of these antibiotic agents to treat biofilm-associated infections (Kandemir et al., 2005). Evidence indicates that 
TABLE 5 | Influence of time on S. aureus biofilm formation.

\begin{tabular}{|c|c|c|c|c|c|c|}
\hline Days & 0 & 1 & 2 & 3 & 4 & 5 \\
\hline $\mathrm{OD}_{630}$ & $0.052 \pm 0.002$ & $0.420 \pm 0.03$ & $0.619 \pm 0.03^{*}$ & $0.625 \pm 0.03^{*}$ & $0.550 \pm 0.03$ & $0.423 \pm 0.02$ \\
\hline
\end{tabular}

$O D_{630}$ means the absorbance of biofilm was measured with a spectrophotometer at $630 \mathrm{~nm}$. *Represent statistical significance $(p \leq 0.05)$.

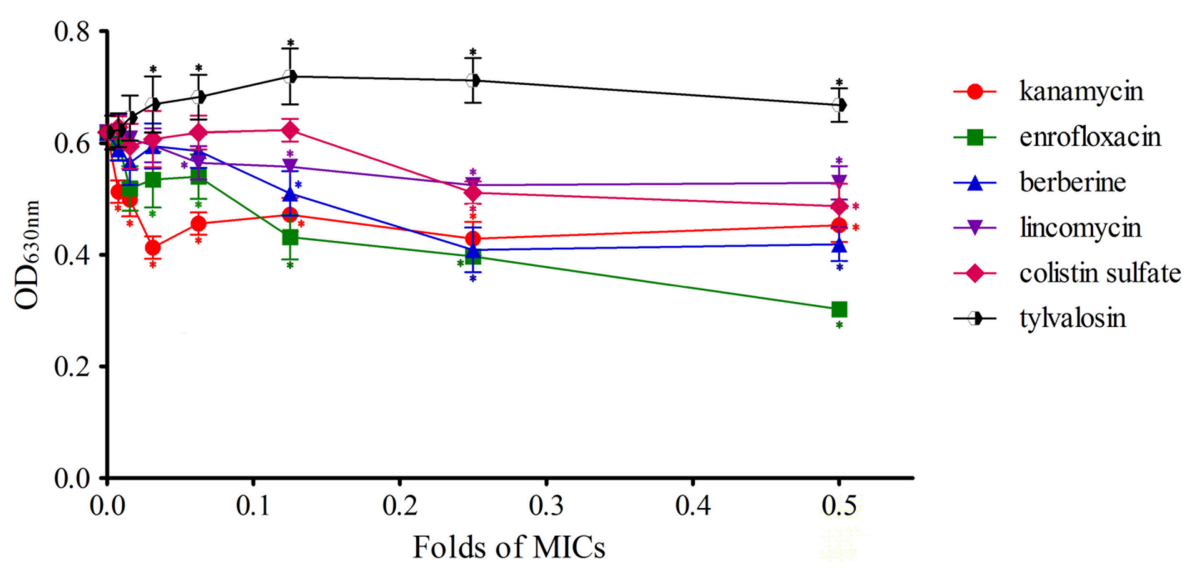

FIGURE 1 | Effects of antimicrobial sub-MIC on S. aureus biofilm formation with crystal violet staining. The biofilms were stained with crystal violet and the optical density was determined with a multi-detection microplate reader at a wavelength of $630 \mathrm{~nm}\left(\mathrm{OD}_{630}\right)$. Data shown are representative three independent experiments and are expressed as mean $\pm \mathrm{SD}$. The treated isolates exhibit a significant reduction in biofilm formation for enrofloxacin, lincomycin, kanamycin, berberine, and colistin sulfate, but not for acetylisovaleryltylosin tartrate. *Represent statistical significance $(p \leq 0.05)$.

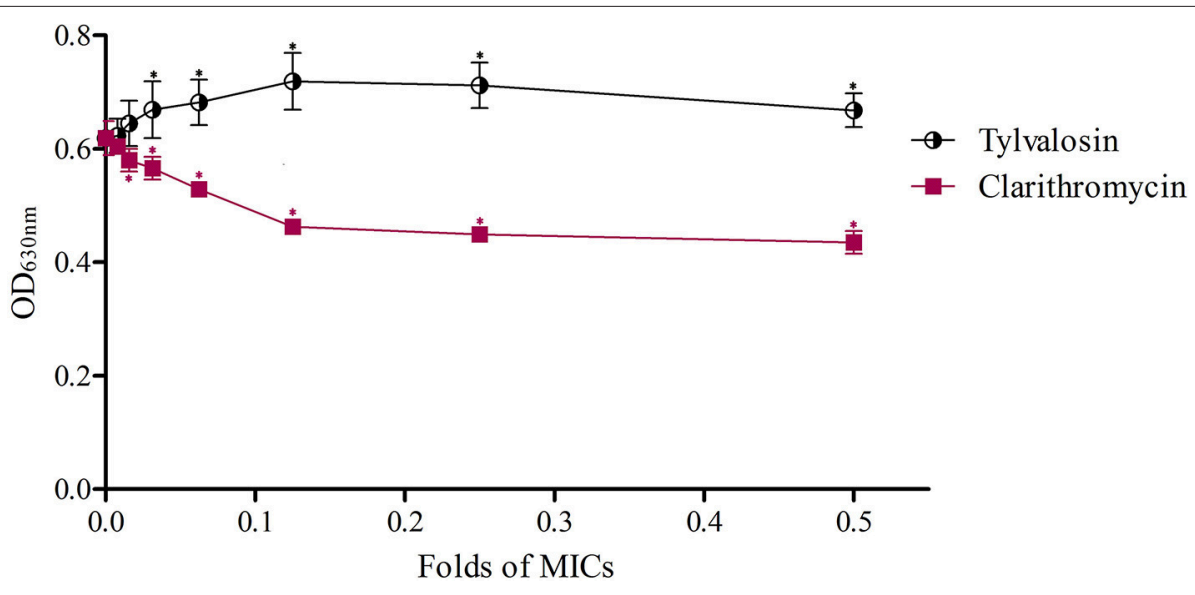

FIGURE 2 | Effects of clarithromycin and acetylisovaleryltylosin tartrate at sub-MIC on S. aureus biofilm formation with crystal violet staining. The biofilms were stained with crystal violet and the optical density was determined with a multi-detection microplate reader at a wavelength of $630 \mathrm{~nm}\left(\mathrm{OD}_{630}\right)$. Data shown are representative three independent experiments and are expressed as mean \pm SD. Sub-MIC of acetylisovaleryltylosin tartrate significantly induced S. aureus biofilm formation but clarithromycin inhibited biofilm formation compared with drug-free bacteria. *Represent statistical significance $(p \leq 0.05)$.

fluoroquinolones used in combination with $\beta$-lactams have a clear synergistic effect against Pseudomonas aeruginosa in vitro (Chachanidze et al., 2009). Our study demonstrated some in vitro interaction and indifference when enrofloxacin was used with $\beta$-lactams against all of the tested bacterial strains. And enrofloxacin in combination with colistin sulfate, sulfadimidine and berberine had the effects of synergy or interaction and antagonism against Gram-negative and Gram-positive bacteria, respectively. For enrofloxacin, the mechanism of action was to antagonize the A subunit of the bacterial enzyme DNA gyrase and thus block the DNA replication (Wolfson and Hooper, 1985). And the bacteriostatic action of sulfanilamide is due to its interference with a hypothetical essential and anti-sulfanilamide metabolite of bacteria (Eyster, 1943). It was not surprising that enrofloxacin in combination with sulfadimidine showed a synergistic effect against $E$. coli and 

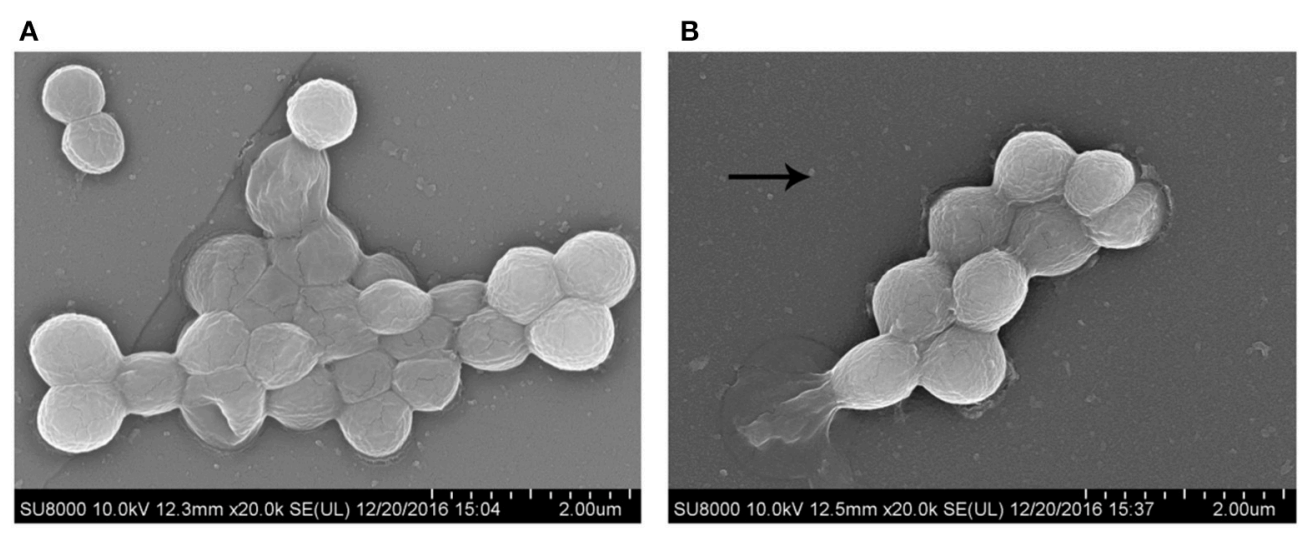

C

D
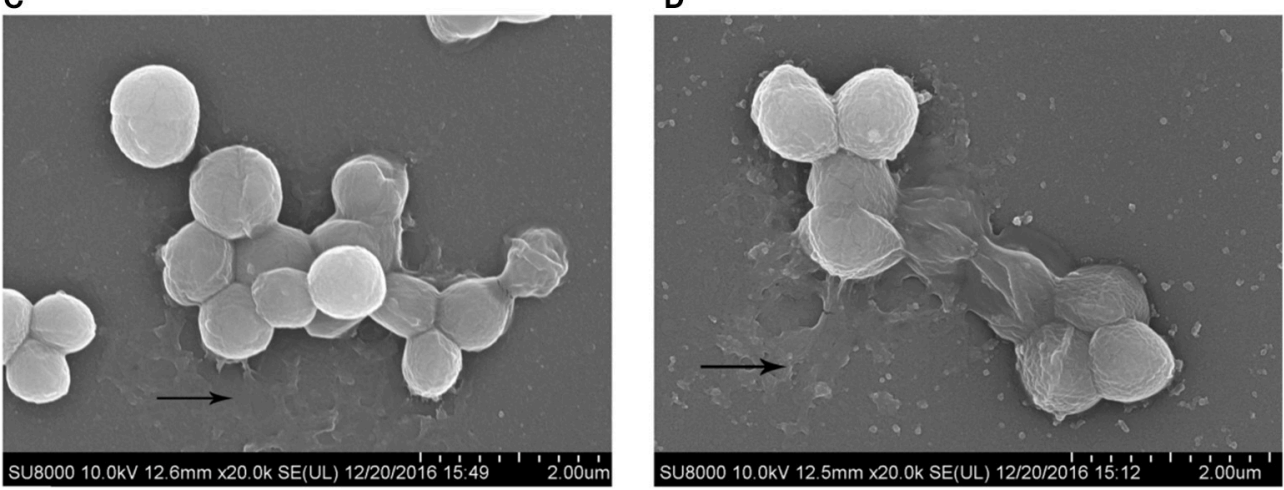

E

$\mathbf{F}$
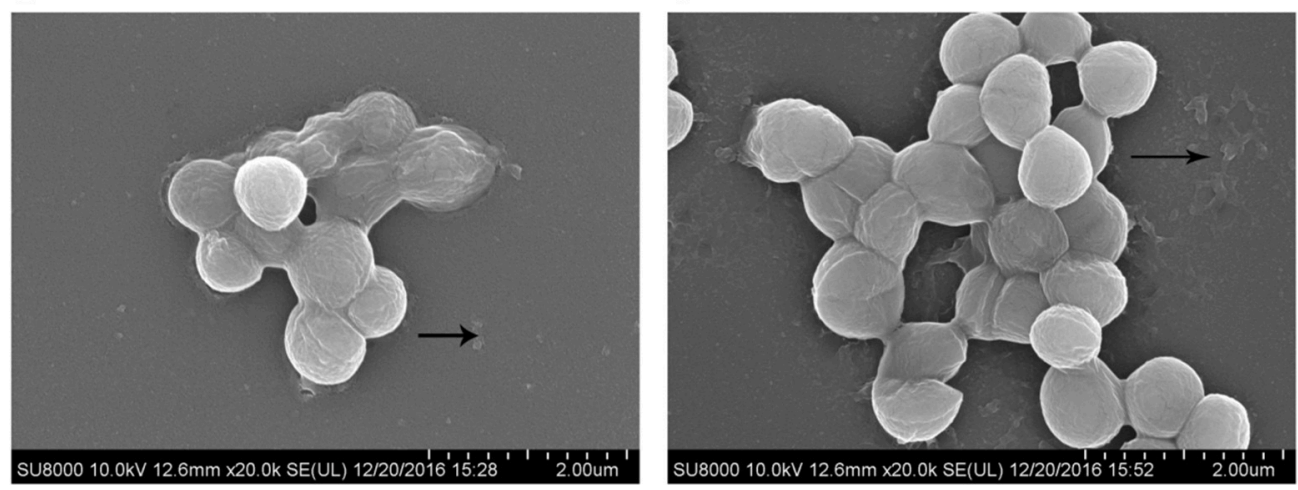

G

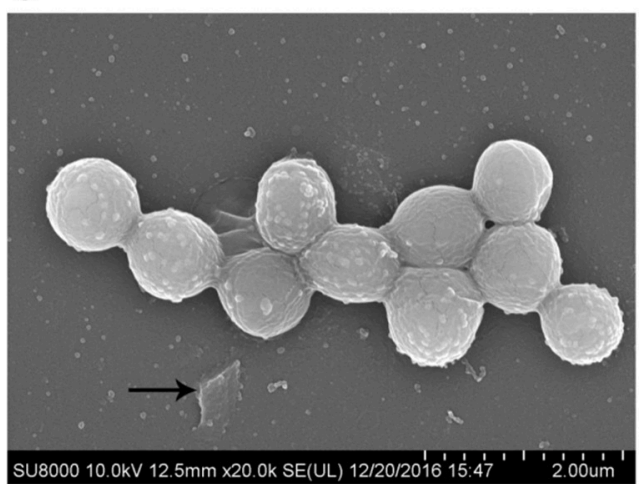

FIGURE 3 | SEM images of biofilm formed by S. aureus incubated with 1/2 MIC of antibiotics for $72 \mathrm{~h}$. Scale bars $=2.00 \mu \mathrm{m}$. (A), drugs-free group; (B), enrofloxacin treatment group; (C), colistin sulfate treatment group; (D), lincomycin treatment group; (E), berberine treatment group; (F), kanamycin treatment group; (G), acetylisovaleryltylosin tartrate treatment group. 


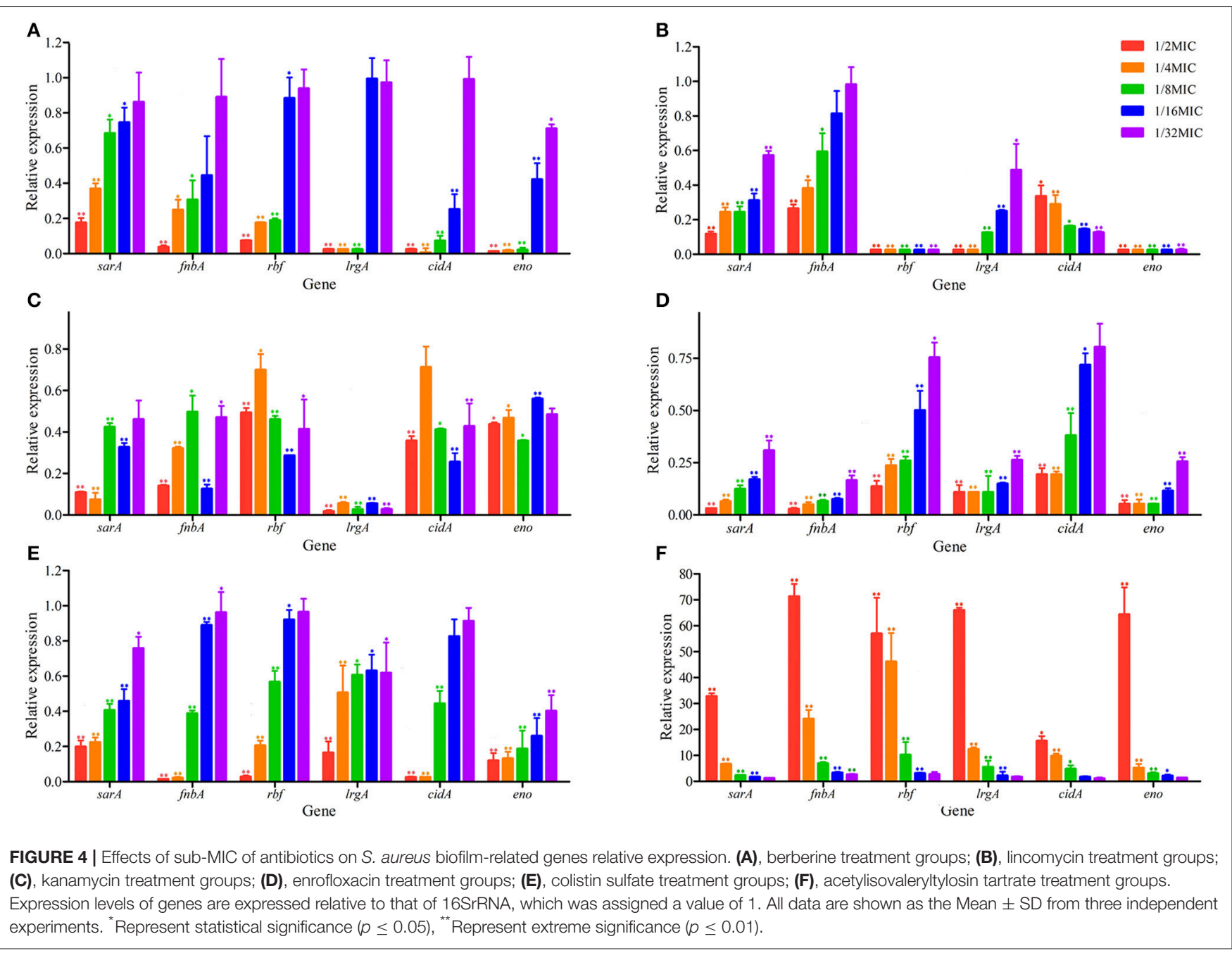

P. multocida. The combination of enrofloxacin with florfenicol against $S$. aureus, E. coli, and $P$. multocida led to antagonistic or indifferent effect, with doxycycline against $S$. aureus showed an interaction and with rifampin against $P$. multocida showed an antagonistic interaction. Rifampin specifically inhibits bacterial RNA polymerase by forming a stable drug-enzyme complex to reduce the efficacy of enrofloxacin (Wehrli, 1983). These results suggest that fluoroquinolones plus $\beta$-lactams, polymyxins, sulfadimidine, and berberine may be alternative combinations for severe infections. Now, rifampin is widely used in clinics and has been proved especially effective in the treatment of tuberculosis (Binda et al., 1971). In the current study, the combination of rifampin with other antimicrobials has been investigated. The combination of rifampin with ceftiofur had the effect of interaction against $S$. aureus, in contrast, which had the effect of indifference against $E$. coli and P. multocida. And rifampin in combination with doxycycline against $S$. aureus and $P$. multocida showed interactive effects. However, when rifampin was combined with kanamycin and lincomycin mainly antagonistic activities were observed. In addition, berberine, traditional medicine, was used to increase its effect on susceptibility when combined with common antibiotics. It has been reported that berberine has weak activity against Gram-negative bacteria and is more active against Grampositive bacteria including $S$. aureus (Vuddanda et al., 2010). In our study, berberine combined with other drugs all exhibited synergy and interaction against Gram-negative bacteria, except for combinations with amoxicillin, florfenicol and lincomycin. Recently, berberine was demonstrated as a strong synergist for many common antibiotics against clinical bacteria (Yu et al., 2005; Zuo et al., 2012). However, the underlying mechanism of the synergistic action of berberine besides bactericidal activities should be a further study.

Regarding the combination susceptibility tests of the current study has been revealed that antibiotics combining against different bacterial strains displayed the different effects. For instance, the indifferent effect between acetylisovaleryltylosin tartrate and berberine was found against $S$. aureus which is sensitive to acetylisovaleryltylosin tartrate and resistant to berberine. the most synergistic effect has been observed for the combination of acetylisovaleryltylosin tartrate and berberine against $P$. multocida, which is all resistant to 
acetylisovaleryltylosin tartrate and berberine. Similarly, colistin sulfate combined with acetylisovaleryltylosin tartrate demonstrated a strong synergic effect against $S$. aureus and $E$. coli in vitro but showed an interactive effect against $P$. multocida. Importantly, the MIC values suggested that E. coli showed a highly resistant to acetylisovaleryltylosin tartrate than $S$. aureus strains, and the strains of E. coli and $P$. multocida are all resistant to acetylisovaleryltylosin tartrate. These data suggested that the combinations of antimicrobial agents that exhibit synergy or partial synergy may augment the antimicrobial effect in resistant strains at a lower concentration. In addition, the combined antimicrobial therapy is usually applied to expand the antimicrobial spectrum and reduce the inhibitory concentration of drug-resistant bacterium.

Current study clearly indicates that the antimicrobial combinations of enrofloxacin plus colistin sulfate or sulfadimidine, rifampin plus colistin sulfate, doxycycline or florfenicol, lincomycin plus colistin sulfate, or sulfadimidine could be used as a remedy for the serious infections caused by a Gram-positive bacterium such as $S$. aureus. In contrast, the combinations of enrofloxacin plus doxycycline, florfenicol or sulfadimidine, rifampin plus kanamycin or florfenicol and lincomycin plus colistin sulfate are not recommended for the treatment of Gram-positive bacterial infections. The combinations of enrofloxacin plus colistin sulfate or sulfadimidine, rifampin plus doxycycline, colistin sulfate or florfenicol, lincomycin plus colistin sulfate or sulfamide are suggested to be used for treating the Gram-negative bacterial infections, such as E. coli and P. multocida. Regarding current results, the combinations of berberine to most of the antibiotics in our tests were found to show a synergistic or interactive effect in addition to amoxicillin, florfenicol and lincomycin.

\section{Effects of Sub-MIC of Antibiotics on S. aureus Biofilm Formation}

Biofilm is a matrix of self-produced extracellular polymeric substances such as exopolysaccharides, proteins, nucleic acids and other substances. And biofilms can play an important role in physical and biological properties such as slow growth or mechanical barrier and in the development of antimicrobial resistance. Staphylococcus aureus is usually used in biofilmrelated trials as it is adept at forming biofilms (Hsu et al., 2011). And crystal violet staining is the most common assay used to qualify biofilms. Until now, there have been very few studies carried out on the effects of sub-MIC of antibiotics on biofilm formation (Hoffman et al., 2005; Dong et al., 2012; Henry-Stanley et al., 2014). While previous studies of the effects of antibiotics on biofilm formation have largely focused on one type of agent at 1/2 MIC, little is known about the use of several antibiotics at $1 / 128 \sim 1 / 2$ MIC (Lee et al., 2014; Silva et al., 2014). In this study, a wide range of subMIC of antimicrobial agents was selected to detect biofilm formation. In our study, we investigated the in vitro effects of sub-MIC of aminoglycosides (kanamycin), fluoroquinolones (enrofloxacin), lincosamides (lincomycin), polypeptides (colistin sulfate), medicinal plant alkaloids (berberine) and macrolides (acetylisovaleryltylosin tartrate) on biofilm formation in S. aureus at $1 / 128 \sim 1 / 2$ MIC. In addition to common antibiotics, we included the plant extract berberine to expand the scope of experimental agents. In addition, biofilm formation in $S$. aureus is the final result of the coordination of many different mechanisms as described in previous reports (Hsu et al., 2011). In this study, the genes $\operatorname{sar} A, f n b A, \operatorname{rbf}, \operatorname{lrg} A, \operatorname{cid} A$, and eno of $S$. aureus were selected to investigate transcription levels using RT-PCR following antibiotic treatment. Recent studies have demonstrated that all genes have a positive effect on $S$. aureus biofilm formation. sarA encodes multiple extracellular proteases, nucleases, and fibronection-binding proteins that play a role in $S$. aureus biofilm formation (Dunman et al., 2001; Cheung et al., 2008; Zielinska et al., 2012). fnbA (fibronectin-binding proteins) encodes proteins to mediate bacterial adherence (Hsu et al., 2011). $\operatorname{lrg} A$ and cidA encode antiholing-like and holing-like proteins, respectively (Rice et al., 2007). And eno encodes laminin-binding proteins (Atshan et al., 2013). The gene $r b f$ is a transcriptional regulator of the $\mathrm{AraC}$ family (Cue et al., 2009). Our morphological observations and crystal violet staining revealed that biofilm formation following sub-MIC treatments of kanamycin, lincomycin, enrofloxacin, colistin sulfate, and berberine was significantly decreased relative to the negative control. But the sub-MIC in the acetylisovaleryltylosin tartrate treatment group had an obvious increase. Furthermore, our RT-PCR results revealed that the following antibacterial treatment, the expression levels of all genes were in agreement with changes in the biofilm.

Previous studies have been reported that aminoglycoside, fluoroquinolone and polypeptide antibiotics all efficiently prevent S. aureus biofilm formation (Saising et al., 2012; Ahire et al., 2013; Hess et al., 2013). The results of our study show that $S$. aureus biofilms incubated with kanamycin, enrofloxacin and colistin sulfate at sub-MIC were significantly inhibited as compared to the control. Hoffman et al. have shown that sub-MIC of aminoglycoside antibiotics induce biofilm formation in $P$. aeruginosa via contributing to aminoglycoside response regulator (Arr) (Hoffman et al., 2005). The results published by Wasfi et al. showed that ciprofloxacin, a fluoroquinolone antibiotic, was found to be the most effective in decreasing $S$. aureus and Enterobacter sp. biofilm formation (Wasfi et al., 2012). Wojnicz et al. also observed that the antibiotics of ciprofloxacin, amikacin, and colistin at subMIC all reduced E. coli biofilm formation in vitro and the inhibitory effects of sub-MIC of agents on biofilm formation was dependent on which antibiotics interfere with the expression of curli fimbriae (Wojnicz and Tichaczek-Goska, 2013).

Data from our study indicated that modest concentration of berberine and lincomycin (1/16-1/2 MIC) were sufficient to inhibit biofilm formation significantly. The lincosamides are known to interact with the 50S ribosomal subunit and affect translation by stimulating peptidyl-tRNA dissociation from the ribosomal particle (Menninger et al., 1994). A study by 
Huang showed that lincosamide antibiotics inhibited biofilm formation in S. aureus (Huang et al., 2012), as did lincomycin in our study. Berberine, a natural bioactive compound, has proven efficacy against microorganisms (Wojtyczka et al., 2014). Chu et al. (2016) have reported that berberine can inhibit MRSA biofilm formation via affecting amyloid fibril formation. Data from Wang et al. investigation indicated that modest concentrations of berberine $(30-45 \mu \mathrm{g} / \mathrm{mL})$ were sufficient to inhibit Staphylococcus epidermidis biofilm formation and the inhibitory effect may be related to the interaction of berberine and bacterial DNA to interfere with the transcription of target genes (Wang et al., 2009). However, the underlying mechanism by which traditional medicines inhibit biofilm formation and enhance antibacterial activity requires further study.

In our study, biofilm formation of $S$. aureus was dosedependently induced by incubation with acetylisovaleryltylosin tartrate. Cirioni et al. found that clarithromycin, a macrolide antibiotic, can effectively prevent $P$. aeruginosa biofilm formation (Cirioni et al., 2011). However, studies by Aka et al. suggested that $P$. aeruginosa biofilm could be induced followed incubation in sub-MIC of azithromycin, a 15-membered macrolide antibiotic (Aka and Haji, 2015). Previous studies have proved that macrolide antibiotics such as 14, 15-membered can inhibit the production of alginate in mucoid strains and the activity of guanosine diphosphomannose dehydrogenase (GMD), the key enzyme in alginate synthesis is decreased, while the 16membered macrolides have no effect on alginate production (Li et al., 2004). Clarithromycin, 14-membered macrolides, can inhibit the formation of $S$. aureus biofilm in our study. Moreover, clarithromycin has been shown to prevent biofilm formation by $S$. aureus (Dicicco et al., 2012). Although controversial, there is increasing evidence for the anti-biofilm properties of Staphylococcal biofilms in vitro (Jabalameli et al., 2012). This indicates that not all antibiotics have inhibited effects on $S$. aureus biofilm formation, even if these antibiotics belong to the same group. However, the underlying mechanisms of acetylisovaleryltylosin tartrate induce biofilm formation are poorly understood and need other investigation and exploration.

As described earlier, biofilm formation is highly associated with antibacterial stimulation. In this study, the effects of subMIC of antibiotics on S. aureus biofilm formation were explored from apparent to genetic. Crystal violet staining was used to investigate the influence of sub-MIC of antibacterial on biofilm formation. And the biofilm changes of $S$. aureus were further investigated using SEM. Consistent with crystal violet staining and morphological observations, the relative expression levels of biofilm-related genes, as determined by qRT-PCR, followed the same trends. Furthermore, the ratio of biofilm formation on a surface was consistent with changes in expression levels between different drug treatment groups. These outcomes suggested that biofilm formation depends on biofilm-related genes and associated proteins under treatment with sub-MIC of antibiotics. And similar results have been reported previously (Bai et al., 2015; Chen et al., 2016). Nevertheless, the mechanisms by which drugs affect biofilm formation should be investigated further.

\section{CONCLUSIONS}

Our data showed synergistic effects of enrofloxacin plus ceftiofur, rifampin plus ceftiofur or doxycycline, berberine plus florfenicol or sulfadimidine against $S$. aureus in vitro. The findings suggest that these reasonable combinations could be used to remedy serious infections caused by Gram-positive bacteria. The antimicrobial effects of enrofloxacin in combination with ceftiofur, colistin sulfate, or sulfadimidine, rifampin in combination with colistin sulfate, doxycycline or florfenicol, lincomycin in combination with colistin sulfate or sulfadimidine on Gram-negative bacteria in vitro are showed addition, what seems to suggest these combinations could be used to remedy Gram-negative bacteria infectious disease. Our study addressed an important clinical concern that reasonable combination of antibiotics may enhance their antibacterial activity and better cure serious and mixed infections.

Results of biofilm formation assays suggest that subMICs of enrofloxacin, lincomycin, kanamycin, colistin sulfate, berberine, and clarithromycin inhibit $S$. aureus biofilm formation. And the biofilm formation was dosedependently inhibited by incubation with berberine and lincomycin, but not enrofloxacin, kanamycin or colistin sulfate, which suggest that sub-MIC of drugs should be selected correct concentrations. However, the sub-MICs of acetylisovaleryltylosin tartrate induce the ability of biofilm formation of $S$. aureus, which indicated that antibiotics belong to the same groups with strains could present different effect on biofilm formation, for instance, subMIC of acetylisovaleryltylosin tartrate induced $S$. aureus biofilm formation but clarithromycin inhibits biofilm formation.

In conclusion, our study offers information on how to select the right antibacterial combinations for the therapy and how to correctly administer the drugs at sub-MIC for preventive use.

\section{AUTHOR CONTRIBUTIONS}

JC conceived and designed the experiments. BY and YZ was responsible for performing the experiments, collecting and analyzing data, and writing the manuscript. ZL, BY, and CW contributed to experiments design, data processing, and experiment performing. SA, SZ, SF, and YQ participated in revised the manuscript. All authors read and approved the final manuscript.

\section{ACKNOWLEDGMENTS}

This work was partly supported by the National Natural Science Foundation of China (grant no. 31572572). 


\section{REFERENCES}

Abdelhady, W., Bayer, A. S., Seidl, K., Moormeier, D., E., Bayles, K., W., Cheung, A. et al. (2014). Impact of vancomycin on sarA-mediated biofilm formation: role in persistent endovascular infections due to methicillin-resistant Staphylococcus aureus. J. Infect. Dis. 209, 1231-1240. doi: 10.1093/infdis/jiu007

Ahire, J. J., Mokashe, N. U., Patil, H. J., and Chaudhari, B. L. (2013). Antioxidative potential of folate producing probiotic Lactobacillus helveticus CD6. J. Food Sci. Technol. 50, 26-34. doi: 10.1007/s13197-011-0244-0

Aka, S. T., and Haji, S. H. (2015). Sub-MIC of antibiotics induced biofilm formation of Pseudomonas aeruginosa in the presence of chlorhexidine. Braz. J. Microbiol. 46, 149-154. doi: 10.1590/S1517-838246120140218

Atshan, S. S., Shamsudin, M. N., Karunanidhi, A., van Belkum, A., Lung, L. T., Sekawi, Z., et al. (2013). Quantitative PCR analysis of genes expressed during biofilm development of methicillin resistant Staphylococcus aureus (MRSA). Infect. Genet. Evol. 18, 106-112. doi: 10.1016/j.meegid.2013.05.002

Bai, J., Wu, Y., Liu, X., Zhong, K., Huang, Y., and Gao, H. (2015). Antibacterial activity of shikimic acid from pine needles of cedrus deodara against staphylococcus aureus through damage to cell membrane. Int. J. Mol. Sci. 16, 27145-27155. doi: 10.3390/ijms161126015

Bharat, A., Martin, I., Zhanel, G. G., and Mulvey, M. R. (2016). In vitro potency and combination testing of antimicrobial agents against Neisseria gonorrhoeae. J. Infect. Chemother. 22, 194-197. doi: 10.1016/j.jiac.2015.10.002

Binda, G., Domenichini, E., Gottardi, A., Orlandi, B., Ortelli, E., Pacini, B., et al. (1971). Rifampicin, a general review. Arzneimittel Forschung 21:1907.

CLSI (2008). Performance Standards for Antimicrobial Disk and Dilution Susceptibility Tests for Bacteria Isolated from Animals; Approved Standard, 3rd Edn., Vol. 28. Wayne, PA: CLSI.

Chachanidze, V., Curbelo-Irizarry, A., Ashcraft, D., and Pankey, G. (2009). In vitro synergy of levofloxacin plus piperacillin/tazobactam against pseudomonas aeruginosa. Interdiscip. Perspect. Infect. Dis. 2009:984934. doi: $10.1155 / 2009 / 984934$

Chen, X., Li, Q., Wang, C., Xu, W., Han, L., Liu, Y., et al. (2016). Prognostic and diagnostic potential of isocitrate dehydrogenase 1 in esophageal squamous cell carcinoma. Oncotarget 7, 86148-86160. doi: 10.18632/oncotarget.13351

Cheung, A. L., Nishina, K. A., Trotonda, M. P., and Tamber, S. (2008). The SarA protein family of Staphylococcus aureus. Int. J. Biochem. Cell Biol. 40, 355-361. doi: 10.1016/j.biocel.2007.10.032

Chu, M., Zhang, M. B., Liu, Y. C., Kang, J. R., Chu, Z. Y., Yin, K. L., et al. (2016). Role of berberine in the treatment of methicillin-resistant Staphylococcus aureus infections. Sci. Rep. 6:24748. doi: 10.1038/Srep24748

Cirioni, O., Ghiselli, R., Silvestri, C., Minardi, D., Gabrielli, E., Orlando, F., et al. (2011). Effect of the combination of clarithromycin and amikacin on Pseudomonas aeruginosa biofilm in an animal model of ureteral stent infection. J. Antimicrob. Chemother. 66, 1318-1323. doi: 10.1093/jac/dkr107

Cue, D., Lei, M. G., Luong, T. T., Kuechenmeister, L., Dunman, P. M., O’Donnell, S., et al. (2009). Rbf promotes biofilm formation by Staphylococcus aureus via repression of icaR, a negative regulator of icaADB. J. Bacteriol. 191, 6363-6373. doi: 10.1128/JB.00913-09

Dicicco, M., Neethirajan, S., Singh, A., and Weese, J. S. (2012). Efficacy of clarithromycin on biofilm formation of methicillin-resistant Staphylococcus pseudintermedius. BMC Vet. Res. 8:225. doi: 10.1186/1746-6148-8-225

Dong, L., Tong, Z., Linghu, D., Lin, Y., Tao, R., Liu, J., et al. (2012). Effects of sub-minimum inhibitory concentrations of antimicrobial agents on Streptococcus mutans biofilm formation. Int. J. Antimicrob. Agents, 39, 390-395. doi: 10.1016/j.ijantimicag.2012.01.009

Donlan, R. M. (2002). Biofilms: microbial life on surfaces. Emerg. Infect. Dis. 8, 881-890. doi: 10.3201/eid0809.020063

Dunman, P. M., Murphy, E., Haney, S., Palacios, D., Tucker-Kellogg, G., Wu, S., et al. (2001). Transcription profiling-based identification of Staphylococcus aureus genes regulated by the agr and/or sarA loci. J. Bacteriol. 183, 7341-7353. doi: 10.1128/jb.183.24.7341-7353.2001

El Haj, C., Murillo, O., Ribera, A., Garcia-Somoza, D., Tubau, F., Cabellos, C., et al. (2016). The anti-biofilm effect of macrolides in a rat model of S. aureus foreignbody infection: might it be of clinical relevance? Med. Microbiol. Immunol. 206, 31-39. doi: 10.1007/s00430-016-0479-5

Eyster, H. C. (1943). Mechanism of sulfanilamide action and its interaction with p-amino benzoic acid. J. Cell. Comp. Physiol. 21, 191-198.
Hall, M. J., Middleton, R. F., and Westmacott, D. (1983). The fractional inhibitory concentration (FIC) index as a measure of synergy. J. Antimicrob. Chemother. $11,427-433$.

Henry-Stanley, M. J., Hess, D. J., and Wells, C. L. (2014). Aminoglycoside inhibition of Staphylococcus aureus biofilm formation is nutrient dependent. J. Med. Microbiol. 63(Pt 6), 861-869. doi: 10.1099/jmm.0.068130-0

Hess, D. J., Henry-Stanley, M. J., Lusczek, E. R., Beilman, G. J., and Wells, C. L. (2013). Anoxia inhibits biofilm development and modulates antibiotic activity. J. Surg. Res. 184, 488-494. doi: 10.1016/j.jss.2013. 04.049

Hoffman, L. R., D’Argenio, D. A., MacCoss, M. J., Zhang, Z., Jones, R. A., and Miller, S. I. (2005). Aminoglycoside antibiotics induce bacterial biofilm formation. Nature 436, 1171-1175. doi: 10.1038/nature03912

Hsu, C. Y., Lin, M. H., Chen, C. C., Chien, S. C., Cheng, Y. H., Su, I. N., et al. (2011). Vancomycin promotes the bacterial autolysis, release of extracellular DNA, and biofilm formation in vancomycin-non-susceptible Staphylococcus aureus. FEMS Immunol. Med. Microbiol. 63, 236-247. doi: 10.1111/j.1574-695X.2011.00846.x

Huang, Q., Yu, H. J., Liu, G. D., Huang, X. K., Zhang, L. Y., Zhou, Y. G., et al. (2012). Comparison of the effects of human beta-defensin 3, vancomycin, and clindamycin on Staphylococcus aureus biofilm formation. Orthopedics 35, e53-e60. doi: 10.3928/01477447-20111122-11

Jabalameli, F., Mirsalehian, A., Khoramian, B., Aligholi, M., Khoramrooz, S. S., Asadollahi, P., et al. (2012). Evaluation of biofilm production and characterization of genes encoding type III secretion system among Pseudomonas aeruginosa isolated from burn patients. Burns 38, 1192-1197. doi: 10.1016/j.burns.2012.07.030

Kandemir, O., Oztuna, V., Milcan, A., Bayramoglu, A., Celik, H. H., Bayarslan, C., et al. (2005). Clarithromycin destroys biofilms and enhances bactericidal agents in the treatment of Pseudomonas aeruginosa osteomyelitis. Clin. Orthop. Relat. Res. 430, 171-175. doi: 10.1097/01.blo.0000137551.45447.89

Lee, J. H., Kim, H. J., and Kim, Y. H. (2017). Is beta-lactam plus macrolide more effective than beta-lactam plus fluoroquinolone among patients with severe community-acquired pneumonia?: a systemic review and meta-analysis. J. Korean Med. Sci. 32, 77-84. doi: 10.3346/jkms.2017.32.1.77

Lee, S., Choi, K. H., and Yoon, Y. (2014). Effect of $\mathrm{NaCl}$ on biofilm formation of the isolate from staphylococcus aureus outbreak linked to ham. Korean J. Food Sci. Anim. Resour. 34, 257-261. doi: 10.5851/kosfa.2014.34.2.257

Li, D., He, L. X., Hu, B. J., Wang, B. Q., Chen, X. H., Li, H. Y., et al. (2004). Effect of azithromycin on the susceptibility of sessile Pseudomonas aeruginosa in biofilm to ceftazidime. Chin. J. Antibiot. 29, 366-370.

Lodise, T. P., Kwa, A., Cosler, L., Gupta, R., and Smith, R. P. (2007). Comparison of beta-lactam and macrolide combination therapy versus fluoroquinolone monotherapy in hospitalized Veterans affairs patients with community-acquired pneumonia. Antimicrob. Agents Chemother. 51, 3977-3982. doi: 10.1128/AAC.00006-07

Majtán, J., Majtánová, L., Xu, M., Majtán, V. (2008). In vitro effect of subinhibitory concentrations of antibiotics on biofilm formation by clinical strains of Salmonella enterica serovar Typhimurium isolated in Slovakia. J. Appl. Microbiol. 104, 1294-1301. doi: 10.1111/j.1365-2672.2007.03653.x

Menninger, J. R., Coleman, R. A., and Tsai, L. N. (1994). Erythromycin, lincosamides, peptidyl-tRNA dissociation, and ribosome editing. Mol. Gen. Genet. 243, 225-233.

Pankey, G. A., and Ashcraft, D. S. (2005). In vitro synergy of ciprofloxacin and gatifloxacin against ciprofloxacin-resistant Pseudomonas aeruginosa. Antimicrob. Agents Chemother. 49, 2959-2964. doi: 10.1128/AAC.49.7.2959-2964.2005

Pankey, G. A., and Ashcraft, D. S. (2009). In vitro antibacterial activity of tigecycline against resistant Gram-negative bacilli and enterococci by time-kill assay. Diagn. Microbiol. Infect. Dis. 64, 300-304. doi: 10.1016/j.diagmicrobio.2009.03.023

Rice, K. C., Mann, E. E., Endres, J. L., Weiss, E. C., Cassat, J. E., Smeltzer, M. S., et al. (2007). The cidA murein hydrolase regulator contributes to DNA release and biofilm development in Staphylococcus aureus. Proc. Natl. Acad. Sci. U.S.A. 104, 8113-8118. doi: 10.1073/pnas.0610226104

Roy, R., Tiwari, M., Donelli, G., and Tiwari, V. (2017). Strategies for combating bacterial biofilms: A focus on anti-biofilm agents and their mechanisms of action. Virulence. doi: 10.1080/21505594.2017.1313372. [Epub ahead of print]. 
Saising, J., Dube, L., Ziebandt, A. K., Voravuthikunchai, S. P., Nega, M., and Götz, F. (2012). Activity of gallidermin on Staphylococcus aureus and Staphylococcus epidermidis biofilms. Antimicrob. Agents Chemother. 56, 5804-5810. doi: 10.1128/aac.01296-12

Silva, J. O., Martins Reis, A. C., Quesada-Gomez, C., Pinheiro, A. Q., Freire, R. S., Oriá, R. B., et al. (2014). In vitro effect of antibiotics on biofilm formation by Bacteroides fragilis group strains isolated from intestinal microbiota of dogs and their antimicrobial susceptibility. Anaerobe 28, 24-28. doi: 10.1016/j.anaerobe.2014.04.010

Stein, C., Makarewicz, O., Forstner, C., Weis, S., Hagel, S., Löffler, B., et al. (2016). Should daptomycin-rifampin combinations for MSSA/MRSA isolates be avoided because of antagonism? Infection 44, 499-504. doi: 10.1007/s15010-016-0874-2

Stewart, P. S., and Franklin, M. J. (2008). Physiological heterogeneity in biofilms. Nat. Rev. Microbiol. 6, 199-210. doi: 10.1038/nrmicro1838

Tiwari, V., Tiwari, D., Patel, V., and Tiwari, M. (2017). Effect of secondary metabolite of Actinidia deliciosa on the biofilm and extra-cellular matrix components of Acinetobacter baumannii. Microb. Pathog. 110, 345-351. doi: 10.1016/j.micpath.2017.07.013

Vuddanda, P. R., Chakraborty, S., and Singh, S. (2010). Berberine: a potential phytochemical with multispectrum therapeutic activities. Expert Opin. Investig. Drugs 19, 1297-1307. doi: 10.1517/13543784.2010.517745

Wang, X., Yao, X., Zhu, Z., Tang, T., Dai, K., Sadovskaya, I., et al. (2009). Effect of berberine on Staphylococcus epidermidis biofilm formation. Int. J. Antimicrob. Agents 34, 60-66. doi: 10.1016/j.ijantimicag.2008.10.033

Wasfi, R., Abd El-Rahman, O. A., Mansour, L. E., Hanora, A. S., Hashem, A. M., and Ashour, M. S. (2012). Antimicrobial activities against biofilm formed by Proteus mirabilis isolates from wound and urinary tract infections. Ind. J. Med. Microbiol. 30, 76-80. doi: 10.4103/0255-0857.93044

Wehrli, W. (1983). Rifampin: mechanisms of action and resistance. Rev. Infect. Dis. 5(Suppl. 3), S407-S411. doi: 10.1093/clinids/5.Supplement_3.S407

Wei, C., Ni, W., Cai, X., Zhao, J., and Cui, J. (2016). Evaluation of Trimethoprim/Sulfamethoxazole (SXT), minocycline, tigecycline, moxifloxacin, and ceftazidime alone and in combinations for SXT-susceptible and SXT-resistant stenotrophomonas maltophilia by In vitro time-kill experiments. PLoS ONE 11:e0152132. doi: 10.1371/journal.pone.0152132

Wojnicz, D., and Tichaczek-Goska, D. (2013). Effect of sub-minimum inhibitory concentrations of ciprofloxacin, amikacin and colistin on biofilm formation and virulence factors of Escherichia coli planktonic and biofilm forms isolated from human urine. Braz. J. Microbiol. 44, 259-265. doi: 10.1590/S1517-83822013000100037

Wojtyczka, R. D., Dziedzic, A., Kepa, M., Kubina, R., Kabała-Dzik, A., Mularz, T., et al. (2014). Berberine enhances the antibacterial activity of selected antibiotics against coagulase-negative Staphylococcus strains in vitro. Molecules 19, 6583-6596. doi: 10.3390/molecules19056583

Wolfson, J. S., and Hooper, D. C. (1985). The fluoroquinolones: structures, mechanisms of action and resistance, and spectra of activity in vitro. Antimicrob. Agents Chemother. 28:581. doi: 10.1128/AAC.28.4.581

Yoshioka, D., Kajiwara, C., Ishii, Y., Umeki, K., Hiramatsu, K., Kadota, J., et al. (2016). Efficacy of beta-lactam-plus-macrolide combination therapy in a mouse model of lethal pneumococcal pneumonia. Antimicrob. Agents Chemother. 60, 6146-6154. doi: 10.1128/AAC.01024-16

Yu, H. H., Kim, K. J., Cha, J. D., Kim, H. K., Lee, Y. E., Choi, N. Y., et al. (2005). Antimicrobial activity of berberine alone and in combination with ampicillin or oxacillin against methicillin-resistant Staphylococcus aureus. J. Med. Food 8, 454-461. doi: 10.1089/jmf.2005.8.454

Zielinska, A. K., Beenken, K. E., Mrak, L. N., Spencer, H. J., Post, G. R., Skinner, R. A., et al. (2012). sarA-mediated repression of protease production plays a key role in the pathogenesis of Staphylococcus aureus USA300 isolates. Mol. Microbiol. 86, 1183-1196. doi: 10.1111/mmi.12048

Zuo, G. Y., Li, Y., Han, J., Wang, G. C., Zhang, Y. L., and Bian, Z. Q. (2012) Antibacterial and synergy of berberines with antibacterial agents against clinical multi-drug resistant isolates of methicillin-resistant Staphylococcus aureus (MRSA). Molecules 17:10322. doi: 10.3390/molecules170910322

Conflict of Interest Statement: The authors declare that the research was conducted in the absence of any commercial or financial relationships that could be construed as a potential conflict of interest.

Copyright (C) 2017 Yang, Lei, Zhao, Ahmed, Wang, Zhang, Fu, Cao and Qiu. This is an open-access article distributed under the terms of the Creative Commons Attribution License (CC BY). The use, distribution or reproduction in other forums is permitted, provided the original author(s) or licensor are credited and that the original publication in this journal is cited, in accordance with accepted academic practice. No use, distribution or reproduction is permitted which does not comply with these terms. 